\title{
Rotational ratchets with dipolar interactions
}

\author{
Sebastian Jäger* and Sabine H. L. Klapp \\ Institute of Theoretical Physics, Technical University Berlin, \\ Hardenbergstr. 36, 10623 Berlin, Germany
}

(Dated: September 24, 2018)

\begin{abstract}
We report results from a computer simulation study on the rotational ratchet effect in systems of magnetic particles interacting via dipolar interactions. The ratchet effect consists of directed rotations of the particles in an oscillating magnetic field, which lacks a net rotating component. Our investigations are based on Brownian dynamics simulations of such many-particle systems. We investigate the influence of both, the random and deterministic contributions to the equations of motion on the ratchet effect. As a main result, we show that dipolar interactions can have an enhancing as well as a dampening effect on the ratchet behavior depending on the dipolar coupling strength of the system under consideration. The enhancement is shown to be caused by an increase in the effective field on a particle generated by neighboring magnetic particles, while the dampening is due to restricted rotational motion in the effective field. Moreover, we find a non-trivial influence of the short-range, repulsive interaction between the particles.

PACS numbers: 82.70.Dd, 05.40.-a, 75.50.Mm
\end{abstract}

\section{INTRODUCTION}

Thermal Brownian ratchets or, as they are sometimes called, Brownian motors, are devices that are able to extract directional motion from Brownian random noise [1]. In these out-of-equilibrium systems, it is possible to rectify the Brownian noise into directional motion. In thermal equilibrium such a phenomenon cannot exist as the second law of thermodynamics would be violated [2, 3].

Thermal ratchet effects have been known for a long time. Recently, however, they are again gaining attention due to their possible applications in biological transport [4, 5] and nanotechnology [6, 7].

Most of the research on Brownian motors has been focused on directed translational motion. Exceptions are recent studies of the so-called rotational ratchet effect in ferrofluids, which has been investigated theoretically [8-10] as well as experimentally [11].

Ferrofluids are suspensions of ferromagnetic colloidal particles (with diameters of about $10 \mathrm{~nm}$ or larger) in a carrier fluid such as water or oil [12, 13]. These systems can be driven out of equilibrium by, e.g., an oscillating magnetic field. The ratchet effect reported in [8, 9] consists of a noise-driven directed rotation of the particles, which are exposed to a field without a net rotating component. The rotations of the particles are associated to an effective torque, which is transferred to the solvent medium. This latter torque is of macroscopic size, making the ferrofluid ratchet effect experimentally detectable [11].

The theoretical investigations so far have been performed on the basis of the single-particle Langevin- and Fokker-Planck equations [9, 10]. Interactions between the ferromagnetic colloids have mostly been neglected [9], the argument being that the concentration of magnetic

* jaeger@itp.tu-berlin.de particles is extremely small in many ferrofluids (volume fraction $\approx 1 \%$ ). In more concentrated samples, however, one would expect the magnetic dipole-dipole interactions between the particles to become important. Indeed, a well known effect is the chain formation of the particles triggered by the anisotropy, particularly the head-to-tail preference, of the dipole interactions. There is, to our knowledge, only one theoretical study in which the impact of the dipolar interactions on the ratchet effect has been investigated [10]. This study approximates the interactions on a mean-field level, i.e., all the particles experience a homogeneous effective field.

In the present paper, we will investigate the impact of the true dipolar interactions on a particle level, i.e., by Brownian dynamics (BD) computer simulations. In this way we can not only capture the full anisotropy and range of the interaction (which is known to be crucial for self-organization processes in dipolar systems [14 16]), but also the fact that the particles are mobile.

This paper is organized as follows: In Sec. II we present the model and the simulation methods used throughout this study. The next section deals with the rotational thermal ratchet effect in non-interacting systems. Here, we will investigate the angular trajectories of the particles and the influence of the strength of the noise and the external field. In Sec. IIIB we will then turn to systems, in which the particles interact via a short-range repulsive and a dipole-dipole potential. We will show that dipolar interactions can enhance as well as suppress the ratchet effect and we will analyze the mechanisms behind these effects. Further, we show that the short-range isotropic, repulsive potential has a significant influence on the ratchet behavior. The paper is then closed with a brief summary and conclusions. 


\section{MODEL AND SIMULATION METHODS}

In this study, we consider a three-dimensional system of dipolar colloidal particles that are immersed in a solvent. Only the dipolar particles are handled explicitly. As a model we use a dipolar soft sphere (DSS) potential, which is comprised of a repulsive part $U^{\text {rep }}$ and a point dipole-dipole interaction part $U^{\mathrm{D}}$ :

$$
U^{\mathrm{DSS}}\left(\mathbf{r}_{i j}, \boldsymbol{\mu}_{i}, \boldsymbol{\mu}_{j}\right)=U^{\mathrm{rep}}\left(r_{i j}\right)+U^{\mathrm{D}}\left(\mathbf{r}_{i j}, \boldsymbol{\mu}_{i}, \boldsymbol{\mu}_{j}\right)
$$

In Eq. (1), $\mathbf{r}_{i j}$ is the vector between the positions of the particles $i$ and $j, r_{i j}$ its absolute value, and $\boldsymbol{\mu}_{i}$ is the dipole moment of the $i$ th particle. The dipolar and repulsive interaction potentials are given by

$$
U^{\mathrm{D}}\left(\mathbf{r}_{i j}, \boldsymbol{\mu}_{i}, \boldsymbol{\mu}_{j}\right)=-\frac{3\left(\mathbf{r}_{i j} \cdot \boldsymbol{\mu}_{i}\right)\left(\mathbf{r}_{i j} \cdot \boldsymbol{\mu}_{j}\right)}{r_{i j}^{5}}+\frac{\boldsymbol{\mu}_{i} \cdot \boldsymbol{\mu}_{j}}{r_{i j}^{3}}
$$

and

$$
U^{\mathrm{rep}}(r)=U^{\mathrm{SS}}(r)-U^{\mathrm{SS}}\left(r_{c}\right)+\left(r_{c}-r\right) \frac{\mathrm{d} U^{\mathrm{SS}}}{\mathrm{d} r}\left(r_{c}\right),
$$

respectively. Here, $U^{\text {rep }}$ is the shifted soft sphere potential, where

$$
U^{\mathrm{SS}}(r)=4 \epsilon\left(\frac{\sigma}{r_{i j}}\right)^{12}
$$

is the unshifted soft sphere (SS) potential for particles of radius $\sigma$.

We investigate the system by making use of Brownian dynamics (BD) simulations. These are based on the translational and rotational Langevin equations [14, 17], which are integrated twice over a time interval that is larger than the inertial relaxation time and small compared to the time on which the configuration changes 18 20]. This procedure results in the equations [19, 21, 22]

$$
\mathbf{r}_{i}(t+\Delta t)=\mathbf{r}_{i}(t)+\frac{1}{k_{B} T} D_{0}^{\mathrm{T}} \mathbf{F}_{i} \Delta t+\sqrt{2 D_{0}^{\mathrm{T}} \Delta t} \boldsymbol{\xi}_{i}^{t}
$$

and

$$
\begin{aligned}
& \mathbf{e}_{i}(t+\Delta t)=\mathbf{e}_{i}(t) \\
& \quad+\frac{1}{k_{B} T} D_{0}^{\mathrm{R}} \mathbf{T}_{i} \Delta t \times \mathbf{e}_{i}(t)+\sqrt{2 D_{0}^{\mathrm{R}} \Delta t} \boldsymbol{\xi}_{i}^{r} \times \mathbf{e}_{i}(t),
\end{aligned}
$$

which form the basis of our BD simulations. Equations (5) and (6) correspond to solving the Langevin equations in the overdamped limit. In the equations above, $\mathbf{e}_{i}=$ $\boldsymbol{\mu}_{i} / \mu$ is the orientation of particle $i$. The conservative forces and torques are given by

$$
\begin{aligned}
\mathbf{F}_{i} & =-\nabla_{\mathbf{r}_{i}} \sum_{j \neq i} U^{\mathrm{DSS}}\left(\mathbf{r}_{i j}, \boldsymbol{\mu}_{i}, \boldsymbol{\mu}_{j}\right) \\
\mathbf{T}_{i} & =\mathbf{T}_{i}^{\mathrm{DSS}}+\mathbf{T}_{i}^{\mathrm{ext}}
\end{aligned}
$$

where

$$
\begin{aligned}
\mathbf{T}_{i}^{\mathrm{DSS}} & =-\boldsymbol{\mu}_{i} \times \sum_{j \neq i} \nabla_{\boldsymbol{\mu}_{i}} U^{\mathrm{DSS}}\left(\mathbf{r}_{i j}, \boldsymbol{\mu}_{i}, \boldsymbol{\mu}_{j}\right) \\
\mathbf{T}_{i}^{\mathrm{ext}} & =\boldsymbol{\mu}_{i} \times \mathbf{B}^{\mathrm{ext}}
\end{aligned}
$$

with an external field $\mathbf{B}^{\mathrm{ext}}$. In Eqs. (5) and (6), $D_{0}^{\mathrm{T}}$ and $D_{0}^{\mathrm{R}}$ are the translational and rotational diffusion constants, which are given by

$$
\begin{aligned}
& D_{0}^{\mathrm{T}}=\frac{k_{B} T}{3 \pi \eta \sigma} \\
& D_{0}^{\mathrm{R}}=\frac{k_{B} T}{\pi \eta \sigma^{3}},
\end{aligned}
$$

where $\eta$ is the viscosity of the solvent. The quantities $\boldsymbol{\xi}_{i}^{t}$ and $\boldsymbol{\xi}_{i}^{r}$ are Gaussian random variables that satisfy

$$
\begin{aligned}
\left\langle\boldsymbol{\xi}_{i}^{t}\right\rangle=0, \quad\left\langle\boldsymbol{\xi}_{j}^{r}\right\rangle & =0 \\
\left\langle\boldsymbol{\xi}_{i}^{t} \boldsymbol{\xi}_{j}^{t}\right\rangle=\boldsymbol{\delta}_{i j}, \quad\left\langle\boldsymbol{\xi}_{i}^{r} \boldsymbol{\xi}_{j}^{r}\right\rangle & =\boldsymbol{\delta}_{i j}, \quad\left\langle\boldsymbol{\xi}_{i}^{r} \boldsymbol{\xi}_{j}^{t}\right\rangle=0
\end{aligned}
$$

Regarding the external field $\mathbf{B}^{\text {ext }}$, we use the same ansatz suggested previously in Refs. [9, 10]. Specifically, the field has a constant component in $x$-direction and an oscillating, yet asymmetric component in $y$-direction. A suitable ansatz is given by

$$
\mathbf{B}^{\mathrm{ext}}(t)=B_{x} \mathbf{e}_{x}+B_{y}\left[\cos \left(\omega_{0} t\right)+\sin \left(2 \omega_{0} t+\delta\right)\right] \mathbf{e}_{y} .
$$

The important point is that this field involves only oscillations, but no full rotations, irrespective of the phase shift $\delta$. Nevertheless, it turns out that the particles can perform directed full rotations. We note that the ansatz (15) is, however, by no means the only field with which a ratchet effect can be realized. In fact a multitude of different fields are suitable, if certain certain conditions are met: $B_{x}$ must be non-vanishing and there cannot be a $\Delta t$ such that $B_{y}(t)=B_{y}(-t+\Delta t$ ) (see Ref. [9] for a detailed discussion of this issue).

Note that the particles we consider here are immersed in a solvent. However, the solvent is only taken into account implicitly and on a single particle level, i.e., the random noise and the diffusion constants do not depend on the configuration of the particles.

We consider $N=500$ particles in our simulation box with periodic boundary conditions. The long-range dipolar interactions are taken into account by using the Ewald summation method [23].

For convenience, we make use of the following reduced units: Field strength $B_{\alpha}^{*}=\left(\sigma^{3} / \epsilon\right)^{1 / 2} B_{\alpha}(\alpha=x, y)$; dipole moment $\mu^{*}=\left(\epsilon \sigma^{3}\right)^{-1 / 2} \mu$; torque $\mathbf{T}^{*}=\mathbf{T} / \epsilon$; time $t^{*}=t D_{0}^{\mathrm{T}} / \sigma^{2}$; temperature $T^{*}=k_{B} T / \epsilon$; position $\mathbf{r}^{*}=\mathbf{r} / \sigma$.

In addition, we will employ the parameter $\lambda=\mu^{* 2} / T^{*}$ measuring the dipolar coupling strength relative to $k_{B} T$. 


\section{RESULTS}

\section{A. The thermal ratchet effect in a non-interacting system}

As a background for our investigation of the impact of dipolar interactions, we discuss in this section BD simulation results for the rotational thermal ratchet effect in systems of non-interacting particles. To this end, we analyze the trajectories of the particles under the influence of the external field (15) as well as the corresponding torque. Further, we investigate the dependence of the ratchet effect on the strength of the external field versus that of the noise. We note that some of the points discussed in this section have already been investigated in Ref. [9] via numerical integration of the respective Fokker-Planck equation. Our present BD results supplement these previous theoretical results.

The systems we consider in this section are characterized by a temperature $T^{*}=0.2$. The particles are driven by a field of frequency $\omega \sigma^{2} / D_{0}^{\mathrm{T}}=15, y$-field component $B_{y}^{*}=1$, and various values of $B_{x}^{*}$. The dipole moment is set to $\mu^{*}=1$, such that the dipole-field coupling is $\mu B_{y} / k_{B} T=\mu^{*} B_{y}^{*} / T^{*}=5$.

Since neither repulsive nor dipolar interactions are taken into account in this section, the density can be chosen arbitrarily. With these choices of the variables, our results are easily comparable to the ones from Ref. [9] (for the precise relations between the dimensionless variables in our study and those in [9], see the Appendix).

To start with, we show in Fig. 1 the mean orientation of the particles,

$$
\overline{\mathbf{S}}(t)=\frac{1}{N} \sum_{i=1}^{N} \mathbf{e}_{i}(t),
$$

for the external field (15) with $B_{x}^{*}=0.1,0.3$ and $\delta=0$. As can be seen, $\bar{S}_{x}$ is essentially constant, while $\bar{S}_{y}$ follows (with a phase lag) the oscillating component of the external field indicated by the dotted line in Fig. 1. Interestingly, while $\bar{S}_{x}$ is increased for the field with $B_{x}^{*}=0.3$ over the field with $B_{x}^{*}=0.1, \bar{S}_{y}$ remains essentially unchanged. We will later see that this is of crucial importance for understanding the impact of interactions.

The behavior of the mean orientation seen in Fig. 1 appears essentially deterministic. The actual ratchet effect is illustrated in Fig. 2. where we plot two angles $\phi_{i}$ and $\bar{\phi}$. The former is the angle that an (arbitrary) particle $i$ encloses with the $x$-axis. It first remains close to a multiple of $2 \pi$ (indicated by the horizontal lines) corresponding to the particle oscillating around the $x$-direction of the field. This behavior continues, until a noise-induced full rotation (i.e., a crossing of a horizontal line) occurs. One also sees that the forward rotation, i.e., an increase by $2 \pi$, occurs more often than the corresponding backward rotation. This illustrates the directional character of the ratchet effect.

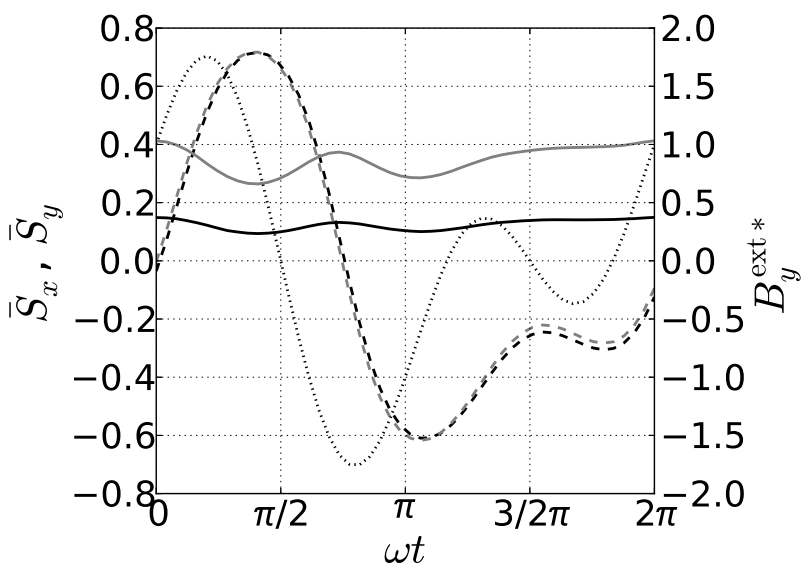

FIG. 1. Mean orientation of the particles during one rotational period of the field for non-interacting systems with $B_{x}^{*}=0.1$ (black lines) and $B_{x}^{*}=0.3$ (gray lines). The $x$ and $y$-components of $\overline{\mathbf{S}}(t)$ are indicated by solid and dashed lines, respectively. The dotted line shows the field component in $y$-direction $B_{y}^{\text {ext* }}$.

The second quantity $\bar{\phi}$ depicted in Fig. 2 corresponds to the averaged value of the angles $\phi_{i}$ of all the particles

$$
\bar{\phi}(t)=\frac{1}{N} \sum_{j=1}^{N} \phi_{j}(t) .
$$

In contrast to $\phi_{i}$ this average angle $\bar{\phi}$ increases monotonically, since individual fluctuations are smeared out.

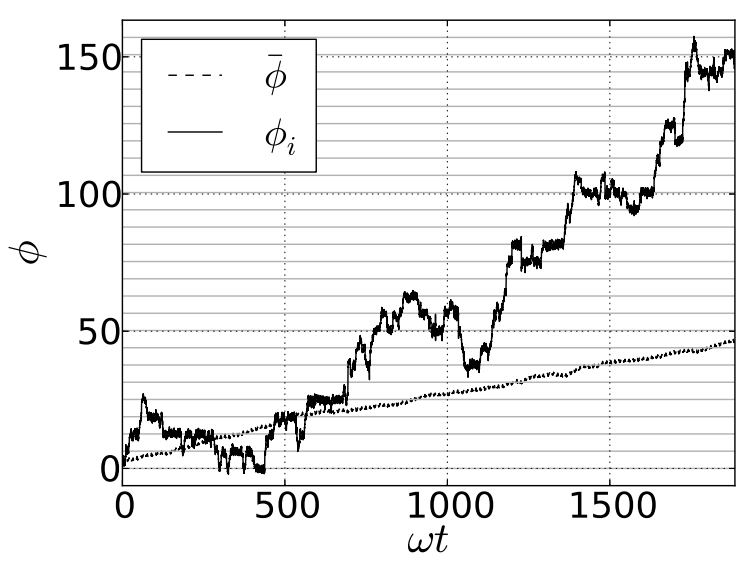

FIG. 2. The angular trajectories in terms of the polar angle $\phi_{i}$ of an individual particle and the system-averaged angle $\bar{\phi}$ at $B_{x}^{*}=0.3$ and $\delta=0$.

Irrespective of these differences between $\phi_{i}$ and $\bar{\phi}$, Fig. 2 clearly demonstrates that there is a net rotational motion in one direction. This corresponds to the presence of a net torque. We calculated the net torque as an 
average of the time-dependent torque over one period of the field, i.e.,

$$
\langle\overline{\mathbf{T}}\rangle=\frac{1}{\tau} \int_{t_{0}}^{t_{0}+\tau} \overline{\mathbf{T}}(t) \mathrm{d} t
$$

where

$$
\overline{\mathbf{T}}(t)=\frac{1}{N} \sum_{i=1}^{N} \boldsymbol{\mu}_{i}(t) \times \mathbf{B}_{i}(t) .
$$

with

$$
\mathbf{B}_{i}=-\nabla_{\boldsymbol{\mu}_{i}} \sum_{j \neq i} U^{\mathrm{DSS}}\left(\mathbf{r}_{i j}, \boldsymbol{\mu}_{i}, \boldsymbol{\mu}_{j}\right)+\mathbf{B}^{\mathrm{ext}} .
$$

Numerical data for the net torque that the particles experience for $B_{x}^{*}=0,0.1$, and 0.3 over the phase difference $\delta$ is presented in Fig. 3 [cf. Eq. (15)]. It is seen that $\left\langle\bar{T}_{z}^{*}\right\rangle$

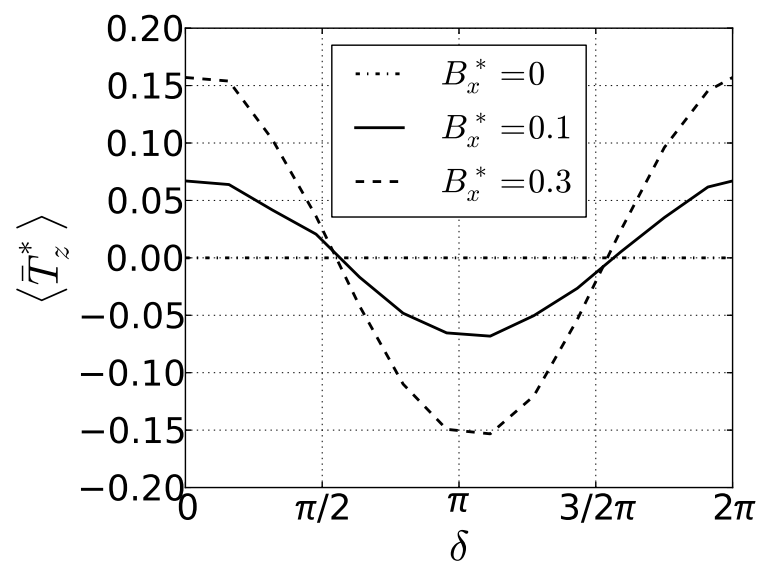

FIG. 3. Averaged torque during one rotational period of the field as function of the phase difference $\delta$ for $B_{x}^{*}=0,0.1$, and 0.3 (and $B_{y}^{*}=1$ ).

is indeed non-zero and (at $\delta=0$ ) positive, reflecting the net rotation of the particles to the right. Increasing the phase difference $\delta$, the value of $\left\langle\bar{T}_{z}^{*}\right\rangle$ changes and even assumes negative values. This implies that the particles can perform forward as well as backward rotations depending on $\delta$.

We now consider in more detail the dependence of the net torque on the strength of the constant field contribution $B_{x}^{*}$. At $B_{x}^{*}=0$, the external field simply performs an oscillation into the $y$-direction. In that case, no net torque can be observed. With only one direction distinguished by the field, directional rotational motion simply cannot occur [2]. For non-vanishing $x$-components the magnitude of $\left\langle\bar{T}_{z}^{*}\right\rangle$ depends strongly on the value of $B_{x}^{*}$. As illustrated by Fig. 3 , increasing $B_{x}^{*}$ from 0.1 to 0.3 results in considerably larger torques $\left\langle\bar{T}_{z}^{*}\right\rangle$. We explain this increase as follows: At higher values of $B_{x}^{*}$, the particles are much more aligned into the $x$-direction of the field, and thus in the plane of the field. The latter point is illustrated by Fig. 1] $\bar{S}_{x}$ is considerably larger for $B_{x}^{*}=0.3$ than for $B_{x}^{*}=0.1$ while $\bar{S}_{y}$ remains essentially unchanged. In other words, higher values of $B_{x}^{*}$ ensure that the particles remain within the plane of the field without dampening the oscillations of the dipole moments in the $y$-direction.

It is well established that ratchet effects, in general, depend strongly on the strength of the noise relative to the deterministic contributions to the equations of motion [2]. For the present system, this interplay is illustrated in Fig. 4. where we plot the torque as a function of the dimensionless temperature $T^{*}$. Inspecting Eq. (6), we see that the temperature $T^{*}$ influences the strength of the deterministic torque (due to the field) alone, if the diffusion constant is kept constant. In other words, $T^{*}$ is a measure for the aforementioned ratio of conservative torques to random noise. Small temperatures correspond to systems that are dominated by deterministic torques, while large temperatures correspond to noise-dominated systems.

In Fig. 4, we can see that the ratchet effect is strongest for finite temperatures in the range $T^{*} \approx 0.05-0.2$. This means that the ratchet effect looses in strength for too small or too large noise contributions. If the temperature is too small, the field dominates the rotational motion of the particles, which are effectively unable to perform rotations against the field. At large temperatures, on the other hand, the noise dominates such that the influence of the field becomes insignificant. However, without the non-equilibrium influence of the external driving field, the ratchet effect cannot exist [2, 3].

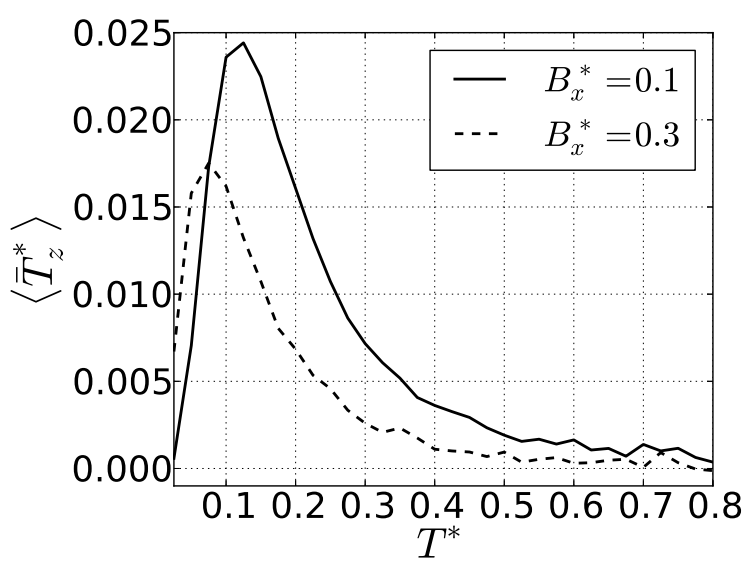

FIG. 4. The net torque as function of the temperature for $B_{x}^{*}=0.1,0.3$.

In Ref. 9], the behavior of a single dipole in the oscillating field (15) was investigated on the basis of a FokkerPlanck equation. Consistent with our results, the authors of Ref. [9] found a maximum in the strength of the net torque at finite values of the noise intensity. Moreover, for the particular choice $B_{x}^{*}=0.3$, our results for the 
mean orientation and the net torque [see Figs. 1 and 3 ] are in quantitative agreement with those in Ref. [9] (see the Appendix for the relation between the dimensionless units).

\section{B. Influence of the particle interactions}

In a ferrofluid, the particles interact with each other via short-range repulsive as well as dipolar interactions. These interactions can be neglected in strongly diluted ferrofluids, they do, however, become important when the density of the dipolar particles becomes higher.

As has been shown in previous studies, particle interactions can indeed have a profound influence on ratchet effects [2, 10, 24]: For instance, in a translational ratchet, they can reverse the direction of the effect or even give rise to it in the first place [2, 25]. The latter is also true for the rotational ratchet effect. It was shown in Ref. [10] that dipolar interactions treated on a simple mean-field level can induce effective particle rotations despite a vanishing field component $B_{x}$.

In the following, we choose a density of $\rho \sigma^{3}=0.2$ corresponding to a dipolar fluid of moderate packing fraction $\eta=\pi \rho \sigma^{3} / 6 \approx 0.105[26]$. This choice ensures that the dipolar interactions play a crucial role at higher coupling strengths. The frequency of the oscillating field is again set to $\omega \sigma^{2} / D_{0}^{\mathrm{T}}=15$. Regarding the interaction parameters, we consider a range of values for the dimensionless dipole moment $\mu^{*}$ and different values of the dimensionless temperature $T^{*}$. In this way we can explore both, impact of the dipolar interactions (2), and those of the repulsive interactions (3).

Note that while we vary $\mu^{*}$ (and, thus, $\lambda$ ), we keep the products $\mu^{*} B_{x}^{*}$ and $\mu^{*} B_{y}^{*}$, i.e., the dipole-field coupling, fixed. To indicate the used field strength, we therefore use the notation $B_{\gamma}^{+} \equiv B_{\gamma}^{*} / \mu^{*}$.

In the lower temperature systems $\left(T^{*}=0.2\right)$ considered here, we use $B_{y}^{+}=1$. With this choice the interaction strength between dipoles and field remains as in the previous section. In the systems with $T^{*}=1$, we use proportionally stronger external fields with $B_{y}^{+}=5$. The relative strength of the external field compared to the Brownian noise is then equal to the one in the low temperature case.

In Fig. 5 we present results for the z-component $\left\langle\bar{T}_{z}^{*}\right\rangle$ of the averaged torque $\left\langle\overline{\mathbf{T}}^{*}\right\rangle$ for systems with different dipolar coupling strengths $\lambda=\mu^{* 2} / T^{*}$ at $B_{x}^{+}=0.5, T^{*}=1$ and $B_{x}^{+}=0.1, T^{*}=0.2$. At $\lambda=0$, the particles interact with each other via the soft-sphere interaction but not via the dipole-dipole interaction. Note that pure softsphere interactions do not affect particle rotations, and thus they should not influence the ratchet effect. Therefore the net torque found at $\lambda=0$ for the $T^{*}=0.2$ system equals the one shown in Fig. 3 for $\delta=0$.

Starting from the non-interacting system, the net torque plotted in Fig. [5] increases up to $\lambda \approx 1.5\left(T^{*}=1\right)$ or $\lambda \approx 2.5\left(T^{*}=0.2\right)$, respectively. In the high tempera- ture system, the maximum of $\left\langle\bar{T}_{z}^{*}\right\rangle$ is approximately $30 \%$ larger than the torque at $\lambda=0$. For $T^{*}=0.2$ the maximum is even more pronounced: The torque is increased by nearly $40 \%$ compared to the non-interacting system. For higher values of $\lambda,\left\langle\bar{T}_{z}^{*}\right\rangle$ decreases continuously.

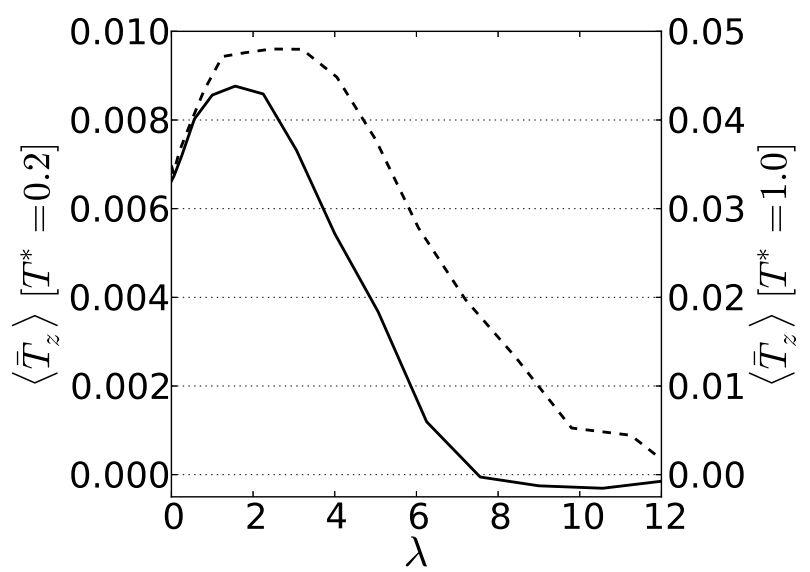

FIG. 5. The z-component $\left\langle\bar{T}_{z}^{*}\right\rangle$ of the averaged torque for different coupling strengths $\lambda$ at $T^{*}=1, B_{x}^{+}=0.5$ (solid line, right axis), and $T^{*}=0.2, B_{x}^{+}=0.1$ (dashed line, left axis).

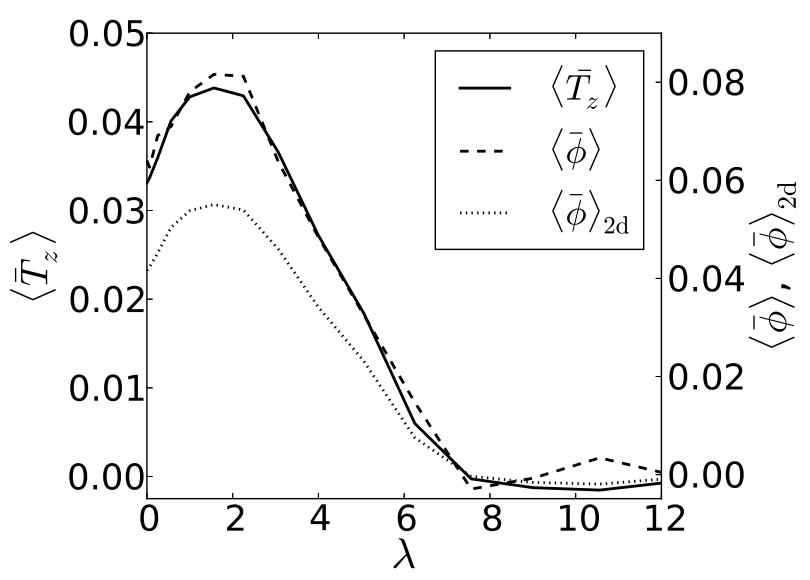

FIG. 6. The net torque $\left\langle\bar{T}_{z}^{*}\right\rangle$, the mean traversed angle $\langle\bar{\phi}\rangle$, and the mean traversed angle $\langle\bar{\phi}\rangle_{2 \mathrm{~d}}$ calculated from the torque [see Eq. [23)] are shown for $T^{*}=1$ and $B_{x}^{+}=0.5$.

The behavior of $\left\langle\bar{T}_{z}^{*}\right\rangle$ is also reflected by the change in the (system-averaged) polar angle $\phi$

$$
\langle\bar{\phi}\rangle=\bar{\phi}(t+\tau)-\bar{\phi}(t)
$$

during one rotational period of the field. As shown in Fig. 6. $\langle\bar{\phi}\rangle$ and $\left\langle\bar{T}_{z}^{*}\right\rangle$ behave almost identically, which can be understood by looking at the $\mathrm{BD}$ evolution equation (6). This equation corresponds to numerically integrat- 
ing

$$
\boldsymbol{\omega}_{i}=\frac{1}{k_{B} T} D_{0}^{\mathrm{R}} \mathbf{T}_{i}+\sqrt{2 D_{0}^{\mathrm{R}}} \boldsymbol{\zeta}_{i} .
$$

Here, $\boldsymbol{\omega}_{i}=\mathbf{e}_{i} \times \dot{\mathbf{e}}_{i}$ is the angular velocity of particle $i$ and $\boldsymbol{\zeta}_{i}$ is a random Gaussian variable. Note that a differential equation for $\dot{\mathbf{e}}_{i}$ can be obtained from Eq. (22) by using the definition of $\boldsymbol{\omega}_{i}$ and taking the vector product with $\mathbf{e}_{i}$. Equation (6) then corresponds to integrating the resulting equation. Assuming the rotational motion of the particles to be restricted to the plane of the field (denoted by the subscript " $2 \mathrm{~d}$ ") and neglecting the random noise, we find

$$
\langle\bar{\phi}\rangle_{2 \mathrm{~d}}=\frac{\tau D_{0}^{\mathrm{R}}}{k_{B} T}\left\langle\bar{T}_{z}\right\rangle_{2 \mathrm{~d}}
$$

from integrating both sides of Eq. (22) over one period of the field. This equation relates the traversed angle of the particles to the average torque. The dotted line in Fig. 6 demonstrates this relation. We calculated the traversed angle via Eq. (23) from the torque component $\left\langle\bar{T}_{z}\right\rangle$. It is seen that this equation slightly underestimates the observed value of $\langle\bar{\phi}\rangle$, which can be explained by the fact that Eq. (23) holds strictly only for particle rotations in the plane of the field. Additionally, we neglected the random noise, which is expected to introduce further deviations from the observed relation. Nonetheless, Eq. (23) captures the general behavior of $\left\langle\bar{T}_{z}\right\rangle$ as function of $\lambda$ quite well.

We now discuss the origin of the maximum in Figs. 5 and 6. The initial increase in strength of the ratchet effect for increasing values of $\lambda$ can be understood by considering the average effective field

$$
\mathbf{B}^{\mathrm{eff}}=\mathbf{B}^{\mathrm{ext}}+\frac{1}{M} \sum_{i=1}^{M} \sum_{j \neq i} \mathbf{B}_{i j}^{\mathrm{dip}}
$$

felt by the particles, where $M$ is the number of particles considered and

$$
\mathbf{B}_{i j}^{\mathrm{dip}}=\frac{3 \mathbf{r}_{i j}\left(\mathbf{r}_{i j} \cdot \boldsymbol{\mu}_{j}\right)}{r^{5}}-\frac{\boldsymbol{\mu}_{j}}{r^{3}} .
$$

This effective field is depicted in Fig. 7 for particles with $\lambda=1.44, T^{*}=1$ and $\lambda=2.45, T^{*}=0.2$. These temperatures and coupling strengths roughly correspond to the maxima in the net torque and the averaged traversed angle [see Fig. 罒. The plots in Fig. 7] show that, due to the dipolar interactions, the effective field components $B_{x}^{\text {eff* }}$ and $B_{y}^{\text {eff* }}$ are increased as compared to the components of the external field. The fact that an enhancement of the (effective) field acting on the particles can support the ratchet effect, is already suggested by our results for a non-interacting system in Sec. IIIA As shown in Figs. 3 and 4 , an increase in $B_{x}$ alone or in both, $B_{x}$ and $B_{y}$, can lead to larger values of the net torque.

For coupling strengths higher than $\lambda \approx 1.5\left(T^{*}=1\right)$ or $\lambda \approx 2.5\left(T^{*}=0.2\right)$, respectively, the magnitude of the

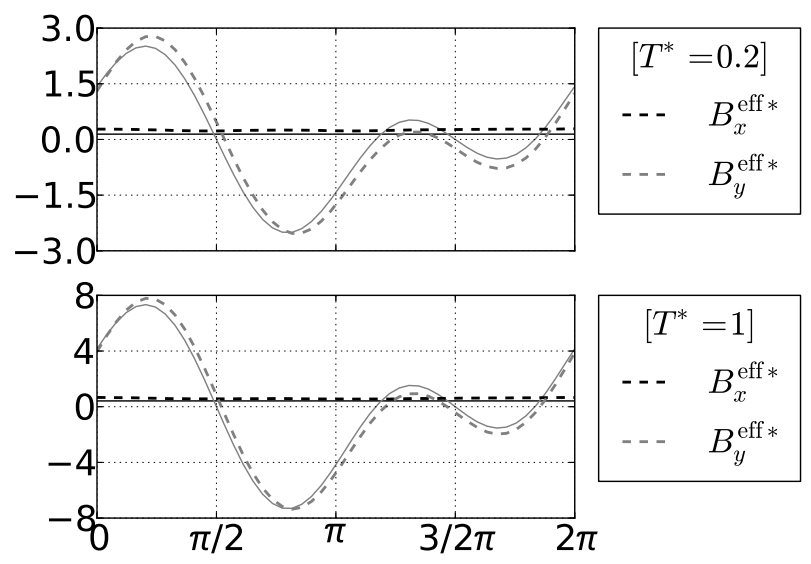

FIG. 7. Mean values of the $x$ - and $y$-components of the averaged effective field $\mathbf{B}^{\text {eff } *}$ over one rotational period of the field at $\lambda=2.45, T^{*}=0.2$, and $B_{x}^{+}=0.1$ (top) and $\lambda=1.44$, $T^{*}=1$, and $B_{x}^{+}=0.5$ (bottom). The solid lines represent the respective external fields, i.e., $B_{x}^{+}=0.1, B_{y}^{+}=1$ (top); $B_{x}^{+}=0.5, B_{y}^{+}=5$ (bottom)

ratchet effect (as measured by $\left\langle\bar{T}_{z}^{*}\right\rangle$ ) begins to decrease. We relate this behavior to the increase of the ratio of conservative torques induced by the dipolar interactions and the external field relative to the strength of the noise. However, in contrast to the (corresponding) decrease described in Sec. IIA [see Fig. 4, a large contribution to the torque now stems from the dipole-dipole interaction and not from the particle-field interaction. This can be seen in Fig. 8, where we compare the functions $\bar{S}_{x}(t)$ and $\bar{S}_{y}(t)\left[\right.$ cf. Eq. (16)] for a system at $\lambda=1.44\left(T^{*}=1\right)$ and a more strongly coupled one at $\lambda=9\left(T^{*}=1\right)$. It is

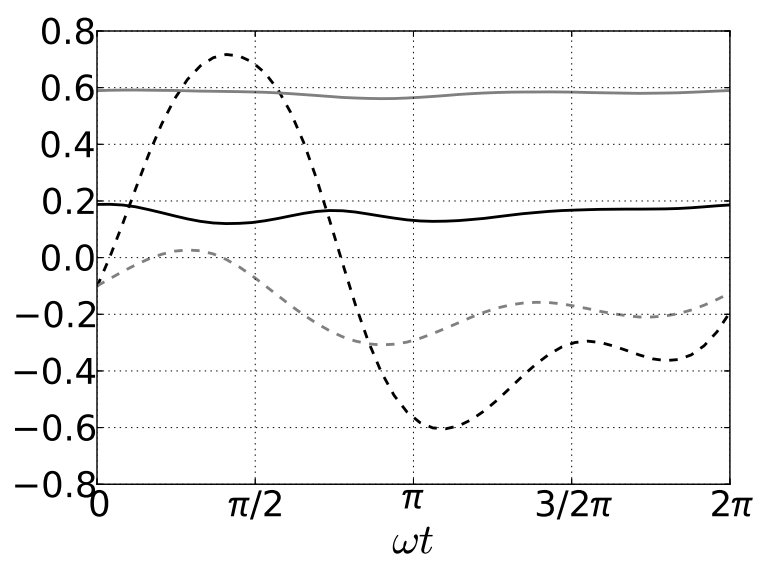

FIG. 8. Mean orientation of the particles during one rotational period of the field for $\lambda=1.44$ (black lines) and $\lambda=9$ (gray lines). The $x$ - and $y$-components of $\overline{\mathbf{S}}$ are indicated by the solid and dashed lines, respectively.

$$
\text { Here, } B_{x}^{+}=0.5 \text { and } T^{*}=1 \text {. }
$$


seen that $\bar{S}_{x}$ is significantly larger for the latter system $\left(\bar{S}_{x} \approx 0.58\right)$ than for the former one $\left(\bar{S}_{x} \approx 0.16\right)$. Recalling the discussion in Sec. III A (non-interacting system), one would thus expect the ratchet effect at $\lambda=9$ to be even larger than at $\lambda=1.44$. However, the amplitude of $\bar{S}_{y}$ is considerably smaller. Indeed, the maximum of $\left|\bar{S}_{y}\right|$ for the strongly coupled system is about 0.31 , while it is about 0.72 for the one with $\lambda=1.44$.

This means that the particles at $\lambda=9$ are much more aligned along the $x$-direction (i.e., the constant part of the field) without closely following the oscillations in $y$ direction. In conclusion, the behavior seen at $\lambda=9$ is in stark contrast to what is shown in Fig. 1 for a noninteracting system. There, an increase in $B_{x}^{*}$ does not automatically damp out the oscillations in the $y$-direction. Consequently, the ratchet effect is increased rather than damped.

Finally, we note that, for relevant values of $\lambda$, the relative increase in the net torque is larger for the lowtemperature system than for the high-temperature one. Indeed, Fig. 5 shows that the value of $T^{*}$ influences the entire behavior of $\left\langle\bar{T}_{z}^{*}\right\rangle$ as a function of $\lambda$. Therefore, not only the dipolar, but also the short-range repulsive interactions between the particles have an impact on the ratchet effect.

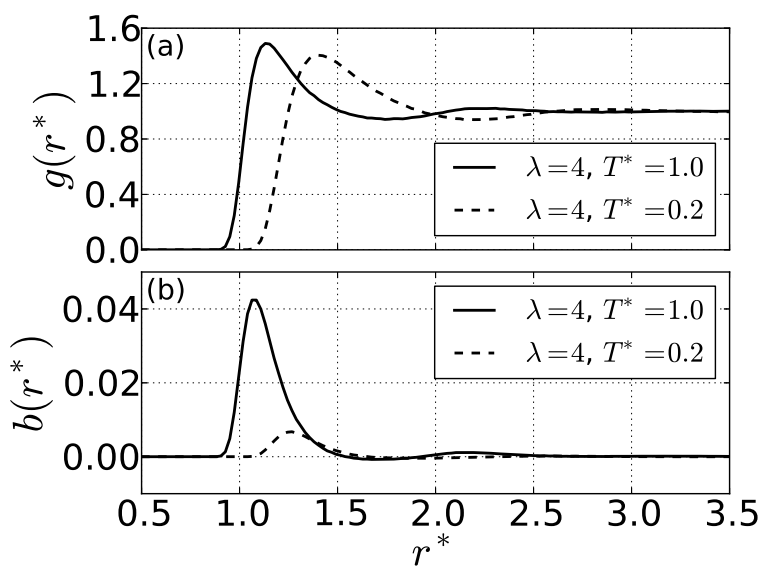

FIG. 9. (a) Pair correlation functions of systems at different temperatures $\left(T^{*}=1,0.2\right)$ but identical dipolar coupling strengths $\lambda$. (b) The local field $b(r)$ [see Eq. (26)] for the two different systems.

The sensitivity against $T^{*}$ can be explained by the fact that the soft-sphere interactions affect the effective distance between the dipolar particles. In Fig 9(a), the radial distribution functions of two systems at identical coupling strength $\lambda=4$ but different temperatures $\left(T^{*}=1\right.$ and 0.2$)$ are shown. Judging from the position of the main peak, two neighbors are typically closer to one another in the $T^{*}=1$ system than in the low temperature one. As a result the particles experience a considerably stronger effective field in the high-temperature system. This is illustrated by Fig. 9(b), where we plot the function

$$
b(r)=\frac{1}{S_{r}} \int_{S_{r}} \mathrm{~d} S \frac{1}{N}\left\langle\sum_{i=1}^{N} \sum_{j \neq i} \delta\left(\mathbf{r}-\mathbf{r}_{i j}\right) B_{x, i j}^{\mathrm{dip}}\right\rangle,
$$

where $S_{r}$ is the surface of a sphere of radius $r$. From a physical point of view, the function $b(r)$ corresponds to the local field in $x$-direction that is generated by neighboring dipolar particles with distance $r^{*}$ from the central one. From Fig. 9(b), we see that the local field at short distances is significantly increased in the $T^{*}=1$ system as compared to the field in the $T^{*}=0.2$ system. Hence, as argued above, the rotational motion is much more restricted in the former system resulting in a less pronounced ratchet effect at a fixed coupling strength.

\section{Relation to self-assembly}

It is well established that strongly coupled dipolar particles can self-assemble into a variety of structures including chains, networks, and sheets [14, 16]. Moreover, for dense systems of dipolar spheres, theory and computer simulations predict a phase with spontaneous long-range, parallel (i.e., ferromagnetic) order [27]. It is therefore an interesting question whether these phenomena have any relevance in the context of the rotational ratchet effect.

The answer from our present BD simulations is essentially negative. Indeed, at the conditions where we found an increase in the ratchet effect $\left(T^{*}=1,0 \lesssim \lambda \lesssim 3.5\right.$; $\left.T^{*}=0.2,0 \lesssim \lambda \lesssim 5.5\right)$ there is no global parallel order. Moreover, significant local ordering of the particles only occurs for dipolar coupling strengths $\lambda \gtrsim 9\left(T^{*}=1\right)$, which is outside of the range where we observe enhancement of the ratchet effect. The structures seen in such a highly coupled systems are illustrated by the simulation snapshot in Fig. 10, Similar to ferrofluids subject to constant, homogeneous external fields, the oscillating field favors chain formation of the particles. Systems of lower coupling strength lack any such order. In particular, no local order can be observed at $\lambda=1.44, T^{*}=1$, i.e., where the ratchet effect is maximal.

Another interesting aspect is the (possible) impact of the ferromagnetic phase transition occurring at higher densities. In Ref. [10], this question was investigated on a mean-field level, where the particles experience a (meanfield) torque of the form

$$
\mathbf{T}_{i}^{\mathrm{mf}}=\mathbf{e}_{i} \times \frac{K}{N} \sum_{i=1, j \neq i}^{N} \mathbf{e}_{j}
$$

where $K$ is the coupling strength. For sufficiently large $K$, one finds a spontaneous ferromagnetic ordering of the particles resulting in an effective non-vanishing field component in $x$-direction [10]. Due to this net field, the ratchet effect can occur even in the absence of an external $x$-component of the field. 


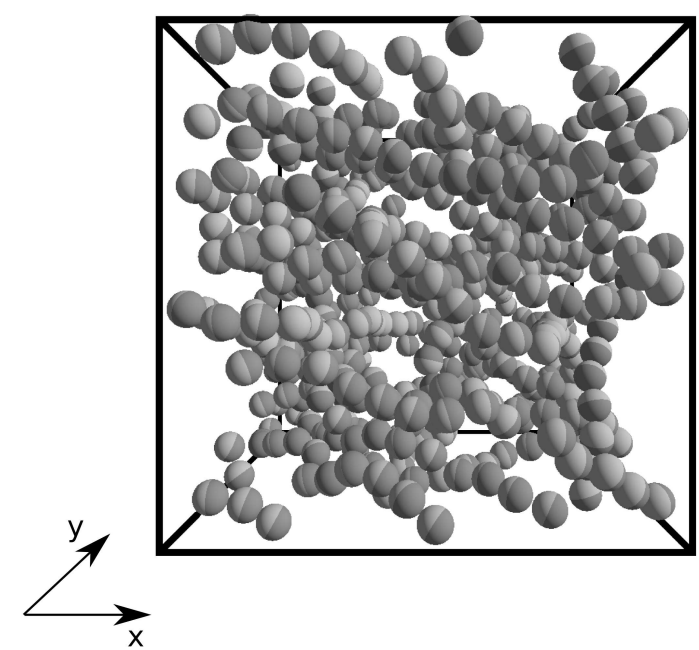

FIG. 10. Snapshot of a system at $\lambda=9, T^{*}=1$, and $B_{x}^{+}=$ 0.5 .

We have searched for a similar phenomenon in our many-particle system. However, at the parameters considered, we were not able to find a net particle rotation. Not even at high densities, where the dipolar soft spheres undergo a ferromagnetic phase transition [27], did we detect such a rotation.

This could be due to several reasons: First, the true effective field within the ferromagnetic phase is inhomogeneous and typically much weaker than any average "mean-field" (this is also the reason that the isotropicferromagnetic transition in a true dipolar system occurs at much larger coupling strengths than those predicted by mean-field theory [10, 27]).

Second, in a dense dipolar system, the orientations of the particles are strongly coupled over large distances. In other words, the dipole orientations are severely restricted, which further suppresses the ratchet effect.

\section{CONCLUSIONS}

In this study, we have investigated the rotational thermal ratchet effect for non-interacting particles as well as particles interacting via long-range dipolar interactions.

With our particle based simulations, we looked at the angular trajectories of the dipolar particles, which conclusively illustrate the net rotating behavior of the driven particles. For non-interacting particles, we found that a finite ratio of deterministic torques to random noise yields a maximally pronounced ratchet effect.

The main focus of this study, however, was the investigation of the influence of dipolar interactions on the rotational behavior of the particles. In particular, we showed that dipolar interactions can have an enhancing as well as a dampening effect depending on the dipolar coupling strength $\lambda$. The enhancement found at small values of $\lambda$ is due to the fact that the effective field acting on a particle is larger (than without interactions), but not too large to suppress rotations. This finding is consistent with the mechanism found in Ref. [10].

Interestingly, we were not able to attribute the increase in the ratchet effect in systems of dipoles to a synchronization phenomenon, i.e., a coupled rotation of two neighboring dipolar particles. It is, however, possible that such synchronization phenomena occur at thermodynamic and field parameters that differ from the ones investigated here.

At higher values of $\lambda$, i.e., stronger dipolar couplings, we find a decrease in the ratchet effect. In this region, the particles start to aggregate into clusters along the direction determined by the constant contribution to the external field. As a consequence, the effective field becomes too strong and the dipole moments can follow the oscillatory motion of the field less and less, leading to a pronounced dampening of any rotations. We note that the values of $\lambda$ considered in this work are in the range typical for real ferrofluids (as are the considered densities).

As a somewhat counterintuitive effect, we have found that not only the anisotropic dipolar interactions but also the isotropic repulsive interactions between the particles have a significant influence on the ratchet effect. At constant dipolar coupling strength, the steepness of these interactions determines the average distance between the particles and thus, the magnitude of the effective local field. In this way, short-range interactions can "tune" the effective torque.

In summary our results show that the conservative interactions typical of real ferrofluids strongly influence noise-induced phenomena such as the ratchet effect. So far, we have not taken into account the fact that the solvent, which is omnipresent in a ferrofluid, induces additional hydrodynamic interactions between the magnetic particles. These long-range interactions have been shown to play a significant role in translational ratchets (see, e.g., Refs. [28, 29]) and related synchronization phenomena [30]. The interplay of hydrodynamic and dipolar interactions in the context of the present ratchet effect will be the subject of a future study.

\section{ACKNOWLEDGMENTS}

We thank A. Engel for the motivation of this work and for helpful discussions. Financial support from the DFG within the RTG 1558 Nonequilibrium Collective Dynamics in Condensed Matter and Biological Systems, project B1, is gratefully acknowledged.

\section{Appendix: Reduced units}

Here, we show how the reduced units used in our study are related to the ones used by Engel et al. [9]. The 
latter are denoted by a superscript " $\nmid$ ". For the reduced temperature we find

$$
T^{*}=\mu_{0}^{*} D^{\dagger} \frac{B^{*}}{B^{\dagger}}
$$

The frequency is related by

$$
\omega^{*}=\frac{3}{D^{\dagger}},
$$

the time by

$$
t^{*}=\frac{1}{3} D^{\dagger} t^{\dagger}
$$

and the torque by

$$
T_{z}^{*}=T_{z}^{\dagger} \frac{T^{*}}{D^{\dagger}}
$$

Using $D^{\dagger}=0.2, \mu_{0}^{*}=1$ [cf. Eq. (10)], and choosing $B^{\dagger}=$ $B^{*}$ and $\Delta t^{\dagger}=0.0015$, yields $T^{*}=0.2, \Delta t^{*}=0.0001$, and $\omega^{*}=15$. This means that $T_{z}^{*}=T_{z}^{\dagger}$.
[1] P. Hänggi and F. Marchesoni, Rev. Mod. Phys. 81, 387 (2009).

[2] P. Reimann, Phys. Rep. 361, 57 (2002).

[3] R. D. Astumian and P. Hänggi, Phys. Today 55, 33 (2002).

[4] M. O. Magnasco, Phys. Rev. Lett. 71, 1477 (1993).

[5] F. Jülicher, A. Ajdari, and J. Prost, Rev. Mod. Phys. 69, 1269 (1997).

[6] J. Rousselet, L. Salome, A. Ajdari, and J. Prost, Nature 370, 446 (1994).

[7] H. Linke, T. E. Humphrey, A. Löfgen, A. O. Sushkov, R. Newbury, R. P. Taylor, and P. Omling, Science 286, 2314 (1999).

[8] A. Engel, H. W. Müller, P. Reimann, and A. Jung, Phys. Rev. Lett. 91, 060602 (2003).

[9] A. Engel and P. Reimann, Phys. Rev. E 70, 051107 (2004).

[10] V. Becker and A. Engel, Phys. Rev. E 75, 031118 (2007).

[11] T. John and R. Stannarius, Phys. Rev. E 80, 050104(R) (2009).

[12] E. Blums, A. O. Cebers, and M. M. Maiorov, Magnetic Fluids (de Gruyter, 1997).

[13] R. E. Rosenzweig, Ferrohydrodynamics (Dover Publications, 1997).

[14] S. Jäger and S. H. L. Klapp, Soft Matter 7, 6606 (2011).

[15] S. Jäger, H. Schmidle, and S. H. L. Klapp, Phys. Rev. E 86, 011402 (2012).
[16] J. Jordanovic, S. Jäger, and S. H. L. Klapp, Phys. Rev. Lett. 106, 038301 (2011).

[17] Z. Wang, C. Holm, and H. W. Müller, Phys. Rev. E 66, 021405 (2002).

[18] J. K. G. Dhont, An Introduction to Dynamics of Colloids (Elsevier, 1996).

[19] D. L. Ermak and J. A. McCammon, J. Chem. Phys. 69, 1352 (1978).

[20] A. J. Banchio and J. F. Brady, J. Chem. Phys. 118, 10323 (2003).

[21] G. Mériguet, M. Jardat, and P. Turq, J. Chem. Phys. 123, 144915 (2005).

[22] E. Dickinson, S. A. Allison, and J. A. McCammon, J. Chem. Soc., Faraday Trans. 2 81, 591 (1985).

[23] J. J. Weis, J. Phys.: Condens. Matter 15, 1471 (2003).

[24] A. Pototsky, A. J. Archer, S. E. Savel'ev, U. Thiele, and F. Marchesoni, Phys. Rev. E 83, 061401 (2011).

[25] P. Reimann, R. Kawai, C. Van den Broeck, and P. Hänggi, Europhys. Lett. 45, 545 (1999).

[26] A. O. Ivanov and O. B. Kuznetsova, Phys. Rev. E 64, 041405 (2001).

[27] S. H. L. Klapp and M. Schoen, J. Chem. Phys. 117, 8050 (2002).

[28] A. Grimm and H. Stark, Soft Matter 7, 3219 (2011).

[29] P. Malgaretti, I. Pagonabarraga, and D. Frenkel, ArXiv e-prints (2012), arXiv:1209.4189 [cond-mat.soft],

[30] J. Kotar, M. Leoni, B. Bassetti, M. C. Lagomarsino, and P. Cicuta, PNAS 107, 7669 (2010). 